\title{
PREDICTORS OF ADHERENCE TO HIV POST-EXPOSURE PROPHYLAXIS AND RETENTION IN CARE AFTER AN EPISODE OF SEXUAL VIOLENCE IN BRAZIL
}

Isabelle Vera Vichr Nisida MD, PhD'; Maria-Ivete Castro Boulos MD'; Lia Maria

Britto da Silva1 , B.S.; Philippe Mayaud MD, PhD ${ }^{1,2}$; Vivian Iida Avelino-Silva MD., PhD ${ }^{1}$; Aluisio Cotrim Segurado MD., PhD. ${ }^{1}$

1Department of Infectious and Parasitic Diseases, Faculdade de Medicina da Universidade de São Paulo, São Paulo, Brazil.

2Faculty of Infectious and Tropical Diseases, London School of Hygiene and Tropical Medicine, London, United Kingdom.

Author disclosure: Authors declare no conflict of interest regarding the publication of the manuscript.

Key words: sexual violence; HIV post-exposure prophylaxis; adherence; retention in care; sexually transmitted infections (STI)

Word count: 3229

Corresponding author: Aluisio C. Segurado

E-mail address: segurado@usp.br

Postal address: Av. Dr. Enéas de Carvalho Aguiar, 470, 05430-000, São Paulo-SP, Brazil

Phone: +5511-30617018; Fax; +5511-30884945 


\begin{abstract}
Adherence to non-occupational post-exposure prophylaxis (nPEP) among sexual violence (SV) victims and their retention in care after SV represent significant challenges. This study aimed at identifying predictors of adherence to nPEP and retention in clinical-laboratory follow-up among SV victims in São Paulo, Brazil. We conducted a retrospective cohort study of SV victims admitted to care and follow-up at the SV unit of the main reference hospital in São Paulo within 72 hours following the SV episode. Eligible patients were submitted to a standardised protocol that included nPEP, screening and management for other STI as well as emergency contraception. Predictors of adherence to nPEP for 28 days and retention in care until discharge at 180 days after admission were analysed. A total of 199 SV episodes in 197 victims were recorded from January/2001 to December/2013 (156 months). Of those episodes, 167 were eligible to receive nPEP and 160 (96\%) actually received a prescription. Overall 104/160 (65\%, 95\%CI 57-72\%) SV victims, who received nPEP, were fully adherent to nPEP up to 28 days, whereas 89/199 (45\%, 95\%CI 38-52\%) were retained in care for 180 days following admission. In multivariate analysis, patients undergoing at least one psychological consultation $(n=126)$ were more likely to adhere to nPEP (adjusted OR 8.32; 95\%CI 3.0-23.3) and to be retained in care for 6 months (adjOR 40.33; 95\%CI 8.33-195.30) compared to patients not receiving psychological support. In contrast, study outcomes were not associated with victims' age and sex and with type of perpetrator. In our cohort provision of psychological care was shown to be associated with enhanced adherence to nPEP and retention in care.
\end{abstract}




\section{INTRODUCTION}

Sexual violence (SV) has been recognized as a public health problem by the World Health Organization since 19941. Because of the use of different definitions, estimates of its worldwide occurrence range between 2-62\% for girls and $3-16 \%$ for boys ${ }^{2}$. Nearly 1 in 5 women (18.3\%) and 1 in 71 men (1.4\%) in the United States have been raped at some time in their lives ${ }^{3}$. In Brazil, the occurrence of SV is estimated to be between 300,000 to 500,000 per year4. Vulnerable groups include women, children and adolescents, people with a history of SV in childhood, individuals under the influence of alcohol and other substances, people with disabilities, those living in conflict areas and low-income individuals with limited access to education and protection by the legal system ${ }^{1-}$ 6.

In the past 20 years, the long-term effects of SV have been more thoroughly studied, particularly the physical and mental health lasting effects, although consequences of SV are not always proportional to the severity of the initial physical trauma experienced by victims. The level of emotional and physical violence or intimidation suffered during the SV episode, the sometimes recurrent nature of exposure, the role of multiple and/or known perpetrators, the early age of the victim, and finally the lack of emotional support provided after the episode, all have an important impact on the short and long-term outcomes of SV1. In this context, lifetime risk of STIs was shown higher after a SV episode ${ }^{7-12}$. Several intertwining factors could mediate risky sexual behavior among SV victims: multiple sexual partners, inconsistent condom use, lack of access to STIs treatment, mental disorders (depression and anxiety), severity of alcohol and illicit drug abuse ${ }^{9-13}$.

Care for SV victims requires a multi-disciplinary approach, including an assessment of emergency medical or surgical issues, such as trauma, screening and prevention of sexually transmitted infections (STI) including HIV, through the provision of non-occupational post-exposure prophylaxis (nPEP); provision of emergency contraception when applicable; and the provision of psychological 
and social support ${ }^{14-17}$. In 2000, a standardized protocol for SV victims was implemented at Hospital das Clinicas, a tertiary reference hospital of the Faculty of Medicine, University of São Paulo: this initiative has encouraged better identification and reporting of SV episodes by the medical emergency services. Globally, adherence to nPEP and retention in care among SV victims have been shown to be limited, ranging from $31,1 \%$ to $51,4 \% 16,17$. In Brazil, whilst nPEP is offered free of charge to eligible SV victims through the national Unified Health System, adherence to completion rarely exceeds $60 \%{ }^{15-19}$. Moreover, there is no evidence of improvement in nPEP adherence among SV victims since this intervention was recommended.

In this study, we investigated predictors of adherence to nPEP for 28 days and of retention in care up to 180 days after inclusion in a cohort of SV victims presenting at a specialized unit in São Paulo, Brazil.

\section{METHODS}

We conducted a retrospective cohort study (January 2001 to December 2013), based on medical chart review of patients admitted to the SV unit of Hospital das Clinicas - a reference tertiary hospital located in the heart of metropolitan area of São Paulo, Brazil -within 72 hours following the SV episode.

\section{Patient and public involvement}

The experience we accumulated in the outpatient care of victims made us develop our research question. Defining predictors for adherence to HIV postexposure prophylaxis (nPEP) and to retention in care after a SV episode was considered a priority to optimize the outcomes of care. The option for a 14-year review of medical charts was based on institutional data availability. The involvement of subjects in the study design or in recruitment of participants was unfeasible, due to the particular confidentiality issues that involve SV. Nevertheless, study results were shared with participants in feedback sessions to emphasize the relevance of their contribution. 


\section{Study procedures}

According to institutional guidelines, upon admission, SV victims are first interviewed and examined by a surgeon or a gynecologist, depending on the type and severity of trauma assessed at entry. Thereafter, they are referred to an infectious disease specialist for collection of detailed information about the SV episode and immediate prescription of nPEP and prophylaxis for other STIs. In addition, they undergo serological screening of HIV, hepatitis B and C, and syphilis (treponemal and non-treponemal tests), as well as emergency contraception if applicable. Genital (vaginal or male urethral) samples for gonoccocal and Chlamydia screening, though recommended in the guidelines, are not systematically collected due to inconsistent availability of laboratory consumables.

HIV nPEP drugs included zidovudine/lamivudine and nelfinavir prior to 2005 and lopinavir/ritonavir thereafter and was considered complete if taken for 28 days. Chemoprophylaxis for STIs includes prevention for trichomoniasis (single dose of oral metronidazole 2g); syphilis and gonorrhea (single dose of intramuscular ceftriaxone 1g); Chlamydia infection (single dose of oral azithromycin 1g); and hepatitis B virus (HBV) active and passive immunization. Emergency contraception is provided whenever penile-vaginal penetration is reported by female victims of fertile age. Screening results are checked at the first outpatient care visit no longer than 7 days after admission, for identification of existing/past STIs. Medication, laboratory tests and multidisciplinary clinical support are provided free of charge, following recommendations of the Brazilian Ministry of Health ${ }^{15}$.

SV victims are then invited to return for follow-up visits on days 7, 30, 90 and 180 after admission, for clinical and laboratory assessment of incident HIV infection and STIs. During follow-up, the comprehensive approach provided to victims and their families also involves nursing care as well as psychological and social worker support. This includes victims and non-offending caregivers counseling, enhanced adherence support to medical interventions by the nursing 
staff, biweekly individual psychodynamic therapy sessions from 2 weeks after the SV episode, tailored according to age for children, adolescents or adults, and appointment with a social worker for assessment of social needs and judicial counseling. Victims are followed up for 180 days to assess seroconversion for HIV, hepatitis B and syphilis, regardless of duration of psychotherapy.

For this study, data retrieved through medical chart review were transferred to a standardized electronic form (REDCap) ${ }^{20}$. The main outcomes variables were: (a) Adherence to nPEP reported at the follow-up visit on day 28; (b) Retention in care, defined as having been retested for HIV and syphilis on day 180. Victims' demographics, clinical aspects, characteristics of the SV episode, physical findings at admission and provision of psychological and social support were assessed as potential predictors of self-reported adherence to nPEP over the next 28 days, and of retention in care during the 180-day follow-up. Psychological support was defined as having attended at least one psychodynamic session, whereas social support was assessed by chart information about family or friend aid.

\section{Statistical analysis}

Variables were described as frequencies with their respective 95\% confidence intervals, medians and interquartile ranges (IQR) as appropriate. Comparisons were performed using Pearson's chi-square test or Fisher's exact test for categorical variables, Wilcoxon-Mann-Whitney rank-sum test for continuous variables, as appropriate. Risk ratios measuring the unadjusted association of potential predictors of adherence to nPEP and retention in care were calculated using tabular methods, along with their respective 95\% confidence intervals (CI). For multivariate analysis, we fitted logistic regression models including variables selected based on causal diagrams and report oddsratios with their 95\%CI. We considered a two-tailed alpha error of 0.05 throughout the analysis, performed using Stata 15.1 (StataCorp College Station, TX: StataCorp LP).

\section{Ethical aspects}


The research protocol was approved by the Institutional Review Board of Hospital das Clinicas (\#0333/11). There was no requirement to ask for retrospective consent of SV victims since data of interest were retrieved from medical charts. Data transposed on CRFs were anonymized and confidentiality was ensured throughout the study.

\section{RESULTS}

Overall, 505 SV victims were seen at the SV unit between January 2001 and December 2013, of whom 251 (50\%) were admitted within a 72-hour window period and eligible for this study. Medical charts were incomplete or unavailable for 52 (20.7\%) SV episodes, leading to a final cohort of 199 SV episodes involving 197 individuals (2 women suffered a second SV episode) for analysis (Figure 1).

SV victims were predominantly female (80\%), white (75\%), of median age 23 (IQR, 17-29) for females and 17 (IQR, 14-32) for males, and included mostly students (46\%) or individuals employed in skilled labor (30\%), with median schooling of 9 years (IQR, 6-11). Mental or physical disability was reported for five (3\%) individuals (Table 1). Median time between the SV episode and admission to the hospital was 1.0 day (IQR, 0.5-1.5). (Table1)

\section{[Table 1]}

Overall, 167 of 199 SV episodes met eligibility criteria for nPEP, but only 160 (96\%) actually received this intervention (3 refusals; 4 did not receive a prescription) (Figure 1). All 199 SV episodes were included in the analysis of predictors of retention in care.

Forty victims were lost from follow-up before day 28, and 16 discontinued nPEP due to misunderstanding of regimen dosage (13 cases) or side effects (3 cases). Among $160 \mathrm{nPEP}$ users, at least one side effect was recorded for 127 cases (79.4\%) and most often included nausea and vomiting 
(31.5\%), diarrhea (26.8\%), neurological disturbances (11.8\%) and skin rashes (2.4\%). Because of side effects, nPEP regimen was modified for $7.1 \%(n=9)$ of victims over the course of treatment.

For 160/199 (85\%) SV episodes, STI prophylaxis was prescribed, including ceftriaxone (85\%), azithromycin (81\%) and metronidazole (76\%). Hepatitis B prophylaxis with immunoglobulin and/or vaccination was provided for 119 (60\%) victims who had not completed vaccination. Emergency contraception was prescribed to $98 / 160$ (61\%) female victims. One woman who did not receive emergency contraception after having reported anal penetration only, eventually got pregnant, and her pregnancy resulted in miscarriage.

Of 160 SV episodes eligible for nPEP prescription, completion of the 28day course was achieved in 104 (65\%, 95\%CI 57-72\%).

Retention in care during the 180-day period was achieved for 89 episodes (45\%, 95\%CI 38-52\%). None of the patients seronegative at baseline had seroconverted for HIV, HBV or hepatitis C infections at the $180^{\text {th }}$ day follow-up assessment. Syphilis seroconversion was detected in a 10-year old girl, who inadvertently missed ceftriaxone prophylaxis.

\section{Predictors of adherence to nPEP}

In univariate analysis (Table 2), we found no statistically significant association between sex, age, race/ethnicity or schooling and adherence to nPEP. Likewise, type of perpetrator (known versus unknown), report of vaginal or anal penetration or of ejaculation at any anatomical site had no significant association with adherence to nPEP. Victims reporting oral penetration $(n=43,27 \%)$ were more likely to adhere to nPEP than those who did not report such an event ( $n=117,73 \%$ ), but this effect did not reach statistical significance (RR1.26, 95\%CI 1.02-1.58; $\mathrm{p}=0.059)$. Social support was associated with higher adherence (72\% vs.47\%, RR 1.52; 95\%CI 0.93-2.47; p=0.03), as was having psychological 
care after SV (RR 2.57; 95\%CI 1.76-3.75; p<0.0001). SV victims reporting SV under the influence of alcohol or other substances were less likely to adhere to nPEP for 28 days (RR 0.68; 95\%CI 0.46-1.02; $\mathrm{p}=0.03$ ).

\section{[Table 2]}

In a multivariate model including sex, age, alcohol or substance use, social support and psychological care, only psychological care retained statistically significant association with adherence to nPEP (adjusted OR 8.32; 95\%CI 3.0023.03; p<0.001) (Table 2)

\section{Predictors of retention in care}

In univariate analysis of predictors of retention in care during 180 days after SV, we failed to find statistically significant associations with sex, age, race/ethnicity or schooling; penetration in oral cavity was associated with higher retention (RR 1.48; 95\%CI 1.09-2.01; $\mathrm{p}=0.019$ )(Table 3).Report of vaginal or anal penetration and of ejaculation at any anatomical site had no significant association with this outcome. As seen in the analysis of predictors of adherence to $\mathrm{nPEP}$, social support (RR 2.01; 95\%CI 1.05-3.87, p=0.011) and psychological care (RR 3.89; 95\%CI 2.22-6.81; $\mathrm{p}<0.001$ ) were associated with higher retention. In contrast, lower retention was observed among patients with physical trauma at admission (RR 0.66; 95\%CI 0.48-0.91; p=0.009) and those reporting SV under the influence of alcohol or other substances (RR 0.45; 95\%CI 0.24-0.85; $\mathrm{p}=0.003)$.

\section{[Table 3]}

We fitted a multivariate model including sex, age, any cavity penetration, physical trauma, social support, psychological care and use of alcohol or other substances as covariates; as in the analysis of independent predictors of nPEP adherence, psychological care persisted as the only independent variable with 
significant association with retention in care (adj OR 40.33, 95\%CI 8.33-195.3, $\mathrm{p}<0.001$ ) (Table 3).

\section{DISCUSSION}

This is the first study to investigate predictors for adherence to nPEP and to retention in care for 180 days in a large cohort of SV victims presenting to emergency departments in Brazil. While adherence to nPEP for 28 days was relatively high (65\%), only $45 \%$ of SV victims were retained in care over 180 days, which is supposed to allow for full assessment of the efficacy of HIV/STI prophylaxis and promotion of health and well-being as well as deployment of social and economic interventions. Provision of psychological care and social support were both associated with higher adherence to nPEP and higher retention in care in univariate analysis, whereas alcohol/substance use prior to or during the SV episode was associated with both lower adherence and lower retention. However, only psychological care remained significantly associated with both outcomes in multivariate analysis.

Knowledge of predictors of adherence to HIV prophylaxis and to retention in care for clinical and laboratory follow-up are useful in the context of SV since more resources can be allocated to those with higher likelihood of withdrawal. Psychological care is an invaluable tool for SV victims, not only due to its direct benefits for emotional welfare, but also as a strategy to improve adherence to medications and retention in care.

Adherence to nPEP in our study was similar to that observed in previous reports from similar Brazilian environments up to 201017-19. Likewise, a systematic review and meta-analysis study showed that the overall adherence to PEP in the context of occupational exposure rarely exceeds $60 \% 21$. Moreover, two meta-analyses on nPEP after SV compared adherence between developed and developing countries 17,22 and reported an overall low adherence to nPEP (40.32\%) among SV victims, although adherence was markedly higher in developing countries than developed ones (53.20\% vs 33.27\%). Authors 
hypothesized that the lower risk of HIV infection in developed countries could be a potential driver of lower prescription and adherence to nPEP17,22. It is also plausible that the availability of specialized SV units and access to nPEP free of charge in some countries, such as Brazil, might also contribute to higher adherence in contrast with others where nPEP depends on payment by the victim or on forensic evidence of SV (e.g.US, Canada;) ${ }^{23}$. However, a clear-cut association between provision of health services to SV victims and adherence to nPEP is still lacking. In Kenya, for instance, Muriuki et al. ${ }^{24}$ recently reported an overall adherence of $34 \%$ to nPEP for 28 days even though antiretroviral were provided free of charge as part of gender-based violence services. In fact, barriers to care, such as the requirement of a police report or forensic examination prior to hospital admission, may also hinder SV victims from seeking health care 25,26 .

Several additional barriers have been described to impair full adherence to nPEP and retention to clinical follow-up after SV. These include factors related to the victims (lack of affordability in case antiretrovirals are not provided for free, drug side effects, emotional barriers, fear of aggressors), as well as structural barriers (lack of public policies and protective services, unavailability of skilled multidisciplinary team support ${ }^{27-29}$ ). Moreover, the way nPEP is prescribed may also affect this outcome. According to a systematic review, provision of a medication start pack does not improve adherence to $\mathrm{PEPP}^{24,30}$. In contrast, qualitative research has shown that inclusion of antiemetic and analgesic drugs in the prescription is beneficial, especially in the first 14 days to reduce the impact of medication side effects, thus contributing to enhance adherence to $\mathrm{nPEP}^{31}$.

Overall, nPEP was highly accepted by our patients, except for 3 (1.8\%) victims. Refusal to take antiretroviral drugs has been reported to occur in up to $14 \%$ of patients, but this rate varies depending on the mode of HIV exposure 21. Digestive complaints were the most common nPEP adverse effects in our cohort, as previously described ${ }^{31}$, but only rarely required discontinuation of chemoprophylaxis. 
Retention in care among SV victims has been previously reported as poor, ranging from 11 to $34.8 \% 19,32$. However, innovative strategies such as a proactive follow-up protocol carried out in South Africa were able to enhance adherence to nPEP to $74 \%$ and retention in care to $81 \% 33$. The protocol included financial incentives for food and transportation and home visits, with victims receiving phone calls prior to follow-up visits and after missed appointments ${ }^{33}$.

Alcohol/substance use prior to or during the SV episode has been reported to occur in 28 to $60 \%$ of cases ${ }^{25,26}$ and is considered a relevant component of rape tactics ${ }^{25}$. In our study, it was reported by only 26 (16\%) victims aged over 14 and was associated with lower adherence to nPEP and with lower retention in care in univariate analysis. However, such an association could not be demonstrated in our multivariate analysis. Our inclusion criteria (admission within $72 \mathrm{~h}$ after the SV episode) may have contributed to reduce the number of alcohol/substance-related SV victims in the cohort. Recognition of the SV episode by the victim has been shown a predictor for seeking medical care and victims of alcohol/substance-related SV are known to be more likely to experience self-blame or to deny the incident ${ }^{34,35}$. In fact, others ${ }^{25,26}$ have highlighted that these individuals are less likely to seek medical care and report to the police after an SV episode.

Victims of substance-related SV have also been shown to exhibit a higher risk of continuing or increasing alcohol abuse after the SV incident, which poses additional challenges for their retention in care ${ }^{25,34}$. Further studies are thus warranted to clarify whether exposure to alcohol/substance prior or during SV delays seeking medical care in the Brazilian context.

Given that $39 \%$ to $70 \%$ of SV victims develop post-traumatic stress syndrome (PTSS), potential benefits of psychological care after SV are expected 8,9 36,37. Cognitive behavior therapy seems to be more effective for adult SV women victims but for children and adolescents more studies are required ${ }^{9,36,37}$. Indeed, 
psychotherapy has been shown to reduce PTSS symptoms when applied early after SV in conflict areas ${ }^{38}$. This could be because acute stress disorder is recognized as a predictor of PTSS after SV37. In our study, we showed that provision of psychological care was an essential element in the management of $\mathrm{SV}$, being associated with enhanced adherence to both nPEP and retention in care. However, we cannot rule out the existence of unmeasured confounders; for example, patients undergoing at least one psychological appointment may have better overall engagement with care.

As far as the methodological approach is concerned, it is important to recognize that our single site retrospective cohort study was based on chart review of patient's or tutor's reports, in the absence of forensic evaluation. Despite the importance of our specialized center in the metropolitan area of Sao Paulo, generalization of our results is limited.

Despite these limitations, the relevance of implementing effective services to respond to the complex needs of SV victims in all settings, including psychological care is unquestionable. In this context, identifying predictors of better outcomes and understanding how to overcome barriers to completion of guidelines may help healthcare providers improve quality of care for SV victims and thus contribute to mitigate the physical and mental health harm of the aggression.

\section{Acknowledgments}

We are grateful to Dr. Carlos Alberto Diegoli, Dr. Fabio Atui, Ms. Marisa do Nascimento and Ms. Silmara Alberguini for their contribution to the comprehensive care provided to sexual victims in this cohort as patients' advisers.

Funding: none received

\section{REFERENCES}

1. Krug EG, Dahlberg LL, Mercy JA et al., eds. World report on violence and health. 
Geneva, World Health Organization 2002; Chapter 6:147-161.

2. Johnson C. Child sexual violence. Lancet 2004;364:462-70.

3. Black MC, Basile KC, Breiding MJ, et al. The National Intimate Partner and Sexual Violence Survey (NISVS): 2010 Summary Report. Atlanta, GA: National Center for Injury Prevention and Control, Centers for Disease Control and Prevention.

4. Cerqueira D, Lima R, Bueno S, et al. Atlas da violência 2018. Publisher: Instituto de Pesquisa Econômica Aplicada (Ipea).2018. Available at: <http://www.ipea.gov.br_atlasviolencia/>. Accessed on 28 March 2019.

5. Garcia-Moreno C, Jansen HAFM, Ellsberg M, et al. WHO multi-country study on women's health and domestic violence against women. Initial results on prevalence, health outcomes and women's responses. World Health Organization, Geneva 2005

6. Basile KC, Breiding MJ, Smith SG. Disability and risk of recent sexual violence in the United States. Am J Public Health 2016;18:1-6.

7. Jenny C, Hooton TM, Bowers et al. Sexually Transmitted Diseases in Victims of Rape. N Engl J Med 1990;322(11):713-6.

.8. Zierler S, Feingold L, Laufer D et al. Adult Survivors of Childhood Sexual Abuse and Subsequent Risk of HIV Infection. Am J Public Health 1991;81(5):5725. 
9. Senn TE, Carey MP, Vanable PA t al .Childhood sexual abuse and sexual risk behavior among men and women attending a sexually transmitted disease clinic. J Consult Clin Psychol 2006;74(4):720-31.

10. Raj A, Silverman JG, Amaro H. The relationship between sexual abuse and sexual risk among high school student: findings from 1997 Massachusetts Youth Risk Behavior Survey. Mater Child Health J 2000 4(2):125-34.

11. Von Sternberg K, Cardoso JB, Jun J et al. An examination of the path between recent sexual violence and sexually transmitted infections. Women's Health Issues 2012;22(3):e283-92.

12. Latimer J, Fleckamn J, Wallace M, Rountree M, Theall K. The influence of violence victimization on sexual health behaviors and outcomes. AIDS Patient Care STDS 2017;31(5):237-244.

13. Humphrey JA and White JW. Women's vulnerability to sexual assault from adolescence to young adulthood. Journal of adolescent health 2000;27: 419-424.

14. Smith DK, Grohskopf LA, Black R, et al. Antiretroviral postexposure prophylaxis after sexual, injection-drug use, or other nonoccupational exposure to HIV in the United States. Recommendations from the U.S. Department of Health and Human Services. MMWR Recomm Rep 2005;54(RR02):1-20.

15. Brasil. Ministério da Saúde. Secretaria de Atenção à Saúde. Departamento de Ações Programáticas Estratégicas Prevenção e tratamento dos agravos 
resultantes da violência sexual contra mulheres e adolescentes. Norma técnica, 3 ed, Brasília: Ministério da Saúde 2012:0-124. Available at:

<http://bvsms.saude.gov.br/bvs/publicacoes/prevencao_agravo_violencia_sexu al_mulheres_3ed.pdf> Accessed on 28 March 2019.

16. WHO. Responding to children and adolescents who have been sexually abused: WHO clinical guidelines. Geneva: World Health Organization; 2017. License: CC BY-NC-SA 3.0 IGO.

17. Chacko L, Ford N, Sbaiti M, et al. Adherence to HIV post-exposure prophylaxis in victims of sexual assault: a systematic review and meta-analysis. Sex Transm Infect 2012;88(5):335-41.

18. Drezett J, Baldacini I, Nisida I, et al. Estudo da adesão à quimioprofilaxia antiretroviral para a infecção por HIV em mulheres sexualmente vitimadas. Rev Bras Ginecol Obstet 1999;21(9):539-44.

19. Garcia MT, Figueiredo RM, Moretti ML, et al. Postexposure prophylaxis after sexual assaults: a prospective cohort study. Sex Transm Dis 2005;32:214-19.

20.Harris PA, Taylor R, Thielke R, et al. Research Electronic Data Capture (REDCap). A metadata driven methodology and workflow process for providing translational research informatics support. J Biomed Inform 2009;42(2):377-81.

21. Ford N, Irvine C, Shubber Z, et al. Adherence to HIV postexposure prophylaxis: A systematic review and meta-analysis. AIDS 2014;28(18):2721-7. 
22. Draughon JE, Sheridan DJ. Nonoccupational postexposure prophylaxis following sexual assault in industrialized low-HIV-prevalence countries: a review. Psychol Health Med 2012;17(2):235-54.

23. Du Mont J, Macdonald, S Myhr T et al. Sustainability of an HIV PEP program for sexual assault survivors: "Lessons Learned" from health care providers. Open AIDS J 2011;5:102-12.

24. Muriuki EM, Kimani J, Machuki Z et al. Sexual assault and HIV postexposure prophylaxis at an urban African Hospital. AIDS Patient Care STDS. 2017 Jun;31(6):255-260.

25. Walsh K, Zinzow HM, Badour CL, et al. Understanding disparities in service seeking following forcible versus drug- or alcohol-facilitated/incapacitated rape. J Interpers Violence 2016;31(14):2475-91.

26. Larsen M-L, Hilden M, Lidegaard O. Sexual assault: a descriptive study of 2500 female victims over a 10-year period. BJOG 2015;122:577-84.

27. Campbell R. The psychological impact of rape victims. Am Psychol 2008;11:702-17.

28. Ullman SE, Filipas HH. Predictors of PTSD symptom severity and social reactions in sexual assault victims. J Trauma Stress 2001;14(2):369-89. 
29. Patel A, Roston A, Tilmon S, et al. Assessing the extent of provision of comprehensive medical care management for female sexual assault patients in US hospital emergency departments. Int J Gynecol Obstet 2013;123(1):24-8.

30.Ford N, Venter F, Irvine C, et al. Starter packs versus full prescription of antiretroviral drugs for postexposure prophylaxis: A systematic review. Clin Inf Dis 2015;60:S182-6.

31.Arend E, Maw A, de Swardt C, et al. South African sexual assault survivors' experiences of post-exposure prophylaxis and individualized nursing care: a qualitative study. J Assoc Nurses AIDS Care 2013;24(2):154-65.

32. Gilles C, Van Loo C, Rozenberg S. Audit on the management of complainants of sexual assault at an emergency department. Eur J Obstet Gynecol Reprod Biol 2010;151(2):185-9.

33. Roland ME, Myer L, Martin LJ, et al. Preventing human immunodeficiency virus infection among sexual assault survivors in Cape Town, South Africa: An observational study. Aids Behav 2012;16(4):990-8.

34. Zinzow HM, Resnick HS, McCauley JL, et al. Prevalence and risk of psychiatric disorders as a function of variant rape histories: Results from a national survey of women. Soc Psychiatry Psychiatr Epidemiol 2012;47(6):893-902.

35. Mason F, Lodrick Z. Psychological consequences of sexual assault. Best Pract 
\& Res Clin Obstet Gynaecol 2013;27(1):27-37.

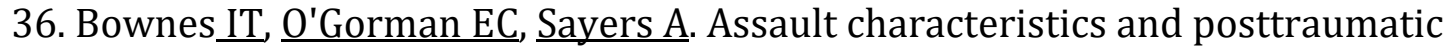
stress disorder in rape victims. Acta Psychiatr Scand 1991;83(1):27-

37. Tiihonen Moller A, Backstrom T, Sondergaard HP, et al. Identifying risk factors for PTSD in women seeking medical help after rape. PLoS ONE 2014;9(10):e111136.

38.Bass JK, Annan J, McIvor Murray S, et al. Controlled trial of psychotherapy for Congolese survivors of sexual violence. N Engl J Med 2013;368(23):2182-91. 
Figure 1: Sexual violence episodes in victims admitted at Hospital das Clinicas and selected to be included in the study, São Paulo, 2001-2013.

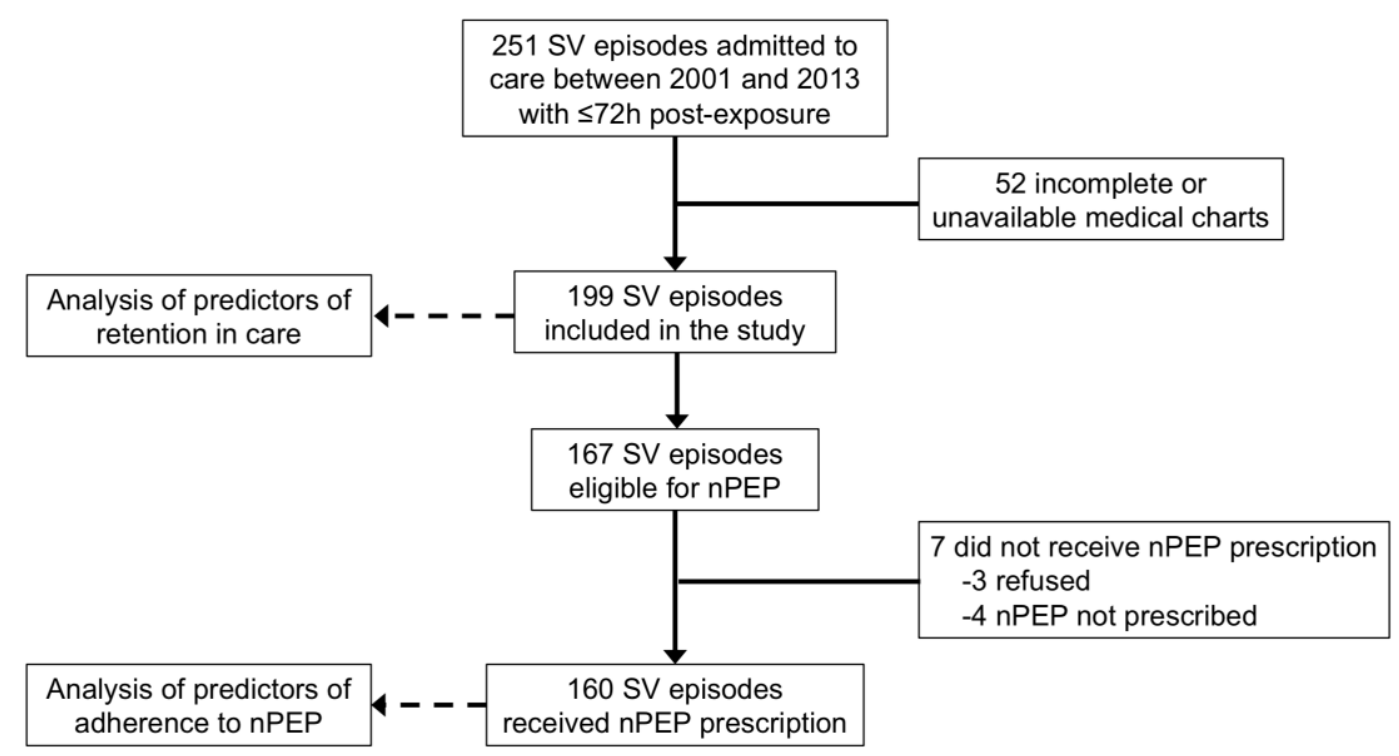


Table 1: Demographic characteristics of 199 sexual violence victims at admission to the emergency room, Hospital das Clinicas, Sao Paulo, 20012013.

\begin{tabular}{lccc}
\hline \multicolumn{1}{c}{ Characteristic } & Overall & Females & Males \\
& n (\%) & n (\%) & n (\%) \\
\hline Number of patients & $199(100)$ & $160(80)$ & $39(20)$ \\
Age (median, IQR) & $22(15-29)$ & $23(17-29)$ & $17(14-32)$ \\
$\leq 13$ years old (\%) & $37(19)$ & $28(17.5)$ & $9(23)$ \\
$14-17$ years old (\%) & $33(17)$ & $20(12.5)$ & $13(33)$ \\
$\geq 18$ years old (\%) & $129(65)$ & $112(70)$ & $17(44)$ \\
Skin color & & & \\
Caucasian (\%) & $149(75)$ & $118(74)$ & $31(79)$ \\
Black/Mixed (\%) & $46(23)$ & $40(25)$ & $6(15)$ \\
Other/Missing (\%) & $4(2)$ & $2(1)$ & $2(5)$ \\
Education years & $9(6-11)$ & $10(7-11)$ & $8(4-11)$ \\
(median, IQR) & & & \\
Occupation & $13(7)$ & $12(8)$ & $1(3)$ \\
Unemployed & $92(46)$ & $69(43)$ & $23(59)$ \\
Student & $22(11)$ & $21(13)$ & $1(3)$ \\
Unskilled labor & $59(30)$ & $49(31)$ & $10(26)$ \\
Skilled labor & $13(7)$ & $9(6)$ & $4(10)$ \\
Unknown & $5(3)$ & $2(1)$ & $3(8)$ \\
Mental or physical & & & \\
disability & & & \\
\hline
\end{tabular}


Table 2: Univariate and multivariate analysis for predictors of adherence to HIV post-exposure prophylaxis (nPEP) among sexual violence victims, Hospital das Clinicas, São Paulo, Brazil, 2001-2013.

\begin{tabular}{|c|c|c|c|c|c|c|}
\hline & \multicolumn{3}{|c|}{ Univariate analysis } & \multicolumn{3}{|c|}{ Multivariate analysis } \\
\hline Predictors & $\mathbf{R R}$ & $95 \% \mathrm{CI}$ & p-value & $\begin{array}{l}\text { Adjusted } \\
\text { OR }\end{array}$ & $95 \% \mathrm{CI}$ & p-value \\
\hline Male sex & 0.99 & $0.74-1.32$ & 0.950 & 0.92 & $0.31-2.71$ & 0.880 \\
\hline $\begin{array}{l}\text { Age (for each 10-year } \\
\text { increase) }\end{array}$ & 1.03 & $0.96-1.11$ & 0.392 & 1.01 & $0.71-1.42$ & 0.976 \\
\hline Caucasian race* & 1.02 & $0.78-1.35$ & 0.874 & & & \\
\hline $\begin{array}{l}\text { Schooling (per year } \\
\text { increase) }\end{array}$ & 1.00 & $0.98-1.01$ & 0.532 & & & \\
\hline Known perpetrator & 0.81 & $0.57-1.15$ & 0.180 & & & \\
\hline Oral penetration & 1.26 & $1.02-1.58$ & 0.059 & & & \\
\hline Vaginal penetration & 0.95 & $0.69-1.30$ & 0.745 & & & \\
\hline Anal penetration & 1.19 & $0.95-1.49$ & 0.138 & & & \\
\hline $\begin{array}{l}\text { Ejaculation (any } \\
\text { anatomical site) }\end{array}$ & 1.02 & $0.78-1.32$ & 0.875 & & & \\
\hline Social support ${ }^{\#}$ & 1.52 & $0.93-2.47$ & 0.036 & 1.41 & $0.40-4.99$ & 0.598 \\
\hline Psychological care & 2.57 & $1.76-3.75$ & $<0.001$ & 8.32 & $3.00-23.03$ & $<0.001$ \\
\hline $\begin{array}{l}\text { Alcohol/substance use at } \\
\text { the time of SV }\end{array}$ & 0.68 & $0.46-1.02$ & 0.030 & 1.29 & $0.41-4.06$ & 0.659 \\
\hline
\end{tabular}

*reference category: non-caucasian; \#includes family, friends and colleagues. 
Table 3: Univariate and multivariate analysis for predictors of retention in care for 180 days among sexual violence victims. Hospital das Clinicas, São Paulo, Brazil, 2001-2013.

\begin{tabular}{|c|c|c|c|c|c|c|}
\hline \multirow[b]{2}{*}{ Predictors } & \multicolumn{3}{|c|}{ Univariate analysis } & \multicolumn{3}{|c|}{ Multivariate analysis } \\
\hline & $\mathbf{R R}$ & 95\%CI & p-value & $\begin{array}{l}\text { Adjusted } \\
\quad \text { OR }\end{array}$ & $95 \% \mathrm{CI}$ & p-value \\
\hline Male sex & 1.04 & $0.71-1.52$ & 0.841 & 0.90 & $0.65-1.24$ & 0.502 \\
\hline $\begin{array}{l}\text { Age (for each 10-year } \\
\text { increase) }\end{array}$ & 0.99 & $0.88-1.11$ & 0.860 & 0.89 & $0.26-3.1$ & 0.855 \\
\hline Caucasian race* & 1.32 & $0.88-1.99$ & 0.152 & & & \\
\hline $\begin{array}{l}\text { Schooling (per year } \\
\text { increase) }\end{array}$ & 1.00 & $0.71-1.31$ & 0.813 & & & \\
\hline Oral penetration & 1.48 & $1.09-2.01$ & 0.019 & & & \\
\hline Vaginal penetration & 1.03 & $0.70-1.52$ & 0.885 & & & \\
\hline Anal penetration & 1.10 & $0.80-1.52$ & 0.558 & & & \\
\hline Penetration(any) & & & & 1.04 & $0.25-4.5$ & 0.952 \\
\hline $\begin{array}{l}\text { Ejaculation (any } \\
\text { anatomical site) }\end{array}$ & 1.09 & $0.78-1.52$ & 0.623 & & & \\
\hline Social support ${ }^{\#}$ & 2.01 & $1.05-3.87$ & 0.011 & 1.53 & $0.36-6.44$ & 0.565 \\
\hline Psychological care & 3.89 & $2.22-6.81$ & $<0.001$ & 40.33 & $8.33-195.3$ & $<0.001$ \\
\hline Physical trauma & 0.66 & $0.48-0.91$ & 0.009 & 0.45 & $0.18-1.14$ & 0.092 \\
\hline $\begin{array}{l}\text { Alcohol/substance use at } \\
\text { the time of SV }\end{array}$ & 0.45 & $0.24-0.85$ & 0.003 & 0.69 & $0.64-2.90$ & 0.613 \\
\hline
\end{tabular}

*reference category: non-caucasian; \#includes family, friends and colleagues. 